\title{
Cyr61/CCN1 Overexpression Induces Epithelial-Mesenchymal Transition Leading to Laryngeal Tumor Invasion and Metastasis and Poor Prognosis
}

\author{
Ying Liu, Yan-Dong Zhou, Yu-Li Xiao, Ming-Hua Li, Yu Wang, Xuan Kan,Qiu- \\ Ying Li, Jian-Guang Lu*, De-Jun Jin*
}

\begin{abstract}
Background: To examine the expression of cysteine-rich 61 (Cyr61/CCN1) protein in laryngeal squamouscell carcinoma (LSCC) tissues, and its relationship with the tumor epithelial-mesenchymal transition (EMT), invasion, metastasis, and prognosis. Materials and Methods: Immunohistochemistry was used to detect the expressions of Cyr61, Vimentin (Vim), and E-cadherin (E-cad) in 88 cases of LSCC tissues and 30 cases of tumor-adjacent normal tissues. Vim and E-cad were used as mesenchymal and epithelial markers, respectively, to determine the relationship between Cyr61 expression and the EMT of LSCC cells. In addition, clinical and histopathological data were combined to analyze the relationship between the positive-expression rates of Cyr61, Vim and E-cad and LSCC invasion, metastasis and prognosis. Results: In LSCC tissues, Vim expression rate was significantly higher than that of the tumor-adjacent tissues, whereas E-cad expression rate was significantly lower than that of the tumor-adjacent tissues. The Vim expression rate was significantly higher in stages T3 and T4 than in stages T1 and T2 LSCC tissues, whereas E-cad expression rate was significantly lower in stages T3 and T4 than in stages T1 and T2 LSCC tissues. Compared to the group without lymph node metastasis, the Vim expression rate was significantly higher and the E-cad expression rate was significantly lower in the group with lymph node metastasis. The expression rate of Cyr61 was significantly higher in LSCC tissues than in the tumor-adjacent normal tissues. In addition, the Cyr61 expression rate was higher in stages $\mathrm{T} 3$ and $\mathrm{T} 4$ than in stages T1 and T2 LSCC, and higher in the group with lymph node metastasis than in the group without lymph node metastasis. The Vim expression rate was significantly higher in the Cyr61 positive group than in the Cyr61 negative group, whereas the E-cad expression rate was significantly higher in the Cyr61 negative group than in the Cyr61 positive group. Survival analysis indicated that survival rates of Cyr61 positive, Vim positive and E-cad negative groups were significantly lower than that of Cyr61 negative, Vim negative and E-cad positive groups, respectively. Conclusions: Cyr61 expression is closely associated with LSCC invasion and lymph node metastasis. Overexpression of Cyr61 may induce EMT and therefore leads to LSCC invasion and metastasis and poor prognosis. Cyr61 may become a new maker for clinical prediction of LSCC invasion and metastasis and a new target for LSCC treatment.
\end{abstract}

Keywords: Cysteine-rich 61 protein - laryngeal squamous-cell carcinoma - immunohistochemistry

Asian Pac J Cancer Prev, 16 (7), 2659-2664

\section{Introduction}

Invasion and metastasis are not only the main characteristics of malignant tumors, but also primary factors for poor prognosis and death in cancer patients. The epithelial-mesenchymal transition (EMT) refers to a biological process involving epithelial cells transforming into cells with mesenchymal phenotypes through a specific program, which plays an important role in embryonic development, chronic inflammation, tissue remodeling, tumor invasion and metastasis, as well as a variety of fibrotic diseases (Baum et al., 2008; Yang et al., 2008; Wang et al., 2013; Grant et al., 2013). EMT is an important biological process for epithelial cell-derived malignant tumor cells to gain the ability of migration and invasion. Through EMT, epithelial cells lose their epithelial phenotypes such as cell polarity and adhesion to the basement membrane, and obtain the abilities of migration, invasion, resistance to apoptosis, and degrading extracellular matrix (ECM), namely "mesenchymal phenotypes" (Yang et al., 2008). Phenotypic changes of the cells also result in changes in the expression of cell markers. For example, the expressions of epithelial markers such as E-cad and keratin decrease, whereas 
the expressions of mesenchymal markers such as Vim, fibronectin, and N-cadherin increase. Therefore, the occurrence of EMT in tumor cells can be determined by monitoring the expression levels of these markers (Chen et al., 2013).

Cyr61/CCN1 is a member of the CCN (CRY61/CTGF/ NOV) family (Hou et al., 2014) and a very important regulatory factor of the ECM, involved in several important physiological and pathological processes such as cell adhesion, migration, proliferation, angiogenesis, inflammatory responses and tissue remodeling (Lau et al., 2011). Cyr61 binds to cell adhesion receptors such as integrins and heparan sulfate proteoglycans (HSPGs) to exert its important functions, including the activation of different downstream pathways, and the regulation of cell adhesion, migration, proliferation, differentiation, apoptosis and survival (Leu et al., 2004; Todorovic et al., 2005). The $\alpha v$ integrin family, especially integrins $\alpha v \beta 3$ and $\alpha v \beta 5$, are indispensable in the expression and secretion of Cyr61 (Crockett et al., 2007; Monnier et al., 2008). Binding of Cyr61 to $\alpha v \beta 3$ and/or $\alpha v \beta 5$ not only contributes to its biological effectiveness, but also further stimulates the secretion of $\alpha v \beta 3$ and $\alpha v \beta 5$, forming the so-called "autocrine loop" (Menendez et al., 2005). More importantly, Cyr61 can interact with growth factors and cytokines, such as TGF- $\beta$ (Bartholin et al., 2007), TNF $\alpha$ (Juric et al., 2012), VEGF (Tsai et al., 2002), BMPs (Su et al., 2010) and the Wnt family members (Sung et al., 2008), and regulate their bioavailabilities and activities, hence broadening their biological roles.

We have demonstrated in the previous study that the $\alpha v$ integrin family proteins are highly expressed in laryngeal or hypopharyngeal squamous cell carcinoma tissues and are closely associated with tumor invasion and metastasis and angiogenesis ( $\mathrm{Lu}$ et al., 2011). The $\alpha \mathrm{v}$ integrin family proteins are a type of RGD receptor and a natural activator of the inactive TGF- $\beta$ precursor. The overexpression of $\alpha \mathrm{v}$ integrins activates the TGF- $\beta$ signaling pathway and triggers a series of subsequent biological responses (Tabatabai et al., 2013). TGF- $\beta$ is one of the main inducing factors of the EMT and is considered as an essential inducing factor in virtually all EMTs occurring in the epithelial tissues (Cichon et al., 2014). Studies also indicated that TGF- $\beta$ overexpression significantly upregulates Cyr61 expression, and there may be an autocrine regulatory loop between the two proteins (Bartholin et al., 2007). Therefore, we speculated that Cyr61 activates the TGF- $\beta$ signaling pathway likely through interacting with $\alpha \mathrm{v}$ integrins, which leads to tumor cells undergoing the EMT and hence tumor invasion and migration as well as poor prognosis.

To our knowledge, studies on the expression and function of Cyr61 in LSCC have not been reported. Therefore, in this study, we used immunohistochemical method to detect the expressions of Cyr61, Vim, and E-cad in LSCC tissues, and found that the expression rate of Cyr61 significantly increased, which was closely associated with LSCC invasion and metastasis and the EMT. Cyr61 overexpression may induce EMT, and therefore promotes LSCC invasion and metastasis, resulting in poor prognosis.

\section{Materials and Methods}

\section{Patients and tissue samples}

Having obtained informed consent and approval by the institutional review board committee, 88 patients ( 69 men and 19 women) with LSCC were included in the study. The patients' age ranged between 40 and 81 years with a median of 56 years. All of the patients had undergone surgery at the Otolaryngology-Head \& Neck Surgery Department of the second affiliated hospital of Harbin Medical University between 2005 and 2008. After surgical resection, the tissues were fixed in $10 \%$ neutral buffered formalin and embedded in paraffin blocks. Sections $(4 \mu \mathrm{m}$ thick) were stained with H\&E. The pathological analysis was done in a blinded manner with respect to the patients' clinical data, and all of the patients were diagnosed to have squamous cell carcinoma. Meanwhile, 30 para-cancerous matched normal tissues derived from the foresaid 88 patients were collected. Using these paraffin-embedded tissue samples, a tissue microarray was constructed by Ao-mei biological technology Co Ltd (Xi'an, China). Histological grading and TNM classification of the patients were done according to the recommendations of the International Union Against Cancer (1997). The primary tumor site: 53 cases of supraglottic cancer, 35 cases of glottic cancers. Histological differentiation: 60 cases of G1, 28 cases of G2\&G3; T stage: 62 cases of T1\&T2 and 26 cases of T3\&T4; N stage: 29 cases with lymph node metastasis $(\mathrm{N}+)$ and 59 cases without lymph node metastasis (N0); M stage: all of the patients were without distant organic metastasis (M0).

\section{Follow-up visit of the patients}

All of the 88 patients were followed for 60 months until December 2013. Thirty-Six patients were identified as death and 9 patients were lost from follow-up data or died of other diseases. The overall survival rate for five years in this group of patients was $59.1 \%$. The lost cases of patients were regarded as censored data and marked on the survival curves.

\section{Immunohistochemical staining}

Four $\mu \mathrm{m}$-thick sections of the tissue microarray were deparaffinized, hydrated and washed with TRIS-buffered saline ( $\mathrm{pH}$ 7.2). After antigen retrieval by microwave pretreatment, endogenous peroxidase activity was blocked by incubation of the slides in water with $0.3 \% \mathrm{H} 2 \mathrm{O} 2$ for $30 \mathrm{~min}$. The sections were then treated with normal goat serum (1/10) for another $30 \mathrm{~min}$ in order to reduce nonspecific binding, followed by incubation overnight at $4^{\circ} \mathrm{C}$ with the primary antibodies, anti-Cyr61/CCN1 (1:50, No. BA1597-1), anti-E-cadherin (1:50, No.BA0474), anti-Vimentin (1:50, No.BM0301), all of these antibodies were purchased from Bioss Co Ltd, Beijing, China. Biotinylated anti-mouse IgG (1:500, Vectastain SP kits, Zhong-shan Co Ltd, Beijing, China) was applied as a secondary antibody for $30 \mathrm{~min}$, followed by application of peroxidase-conjugated streptavidin for $30 \mathrm{~min}$. The immune reaction was revealed with diaminobenzidine tetrachloride. Consistent negative controls were obtained by omission of the primary antibody. The results of the 
immunohistochemical staining were evaluated separately by two investigators in a blinded manner. The percentage of positive tumor cells was determined by examining at least five representative microscope areas at campus visualize of 200 magnifications. The staining was scored as negative (-) if $<5 \%$ demonstrated staining, intermediate positive (+) for staining between $5-50 \%$, and strong positive $(++)$ for staining $>50 \%$.

\section{Statistical analysis}

Statistical analysis was done using the SPSS software program version 17.0. $\chi 2$ test was used to establish the statistical significance of the expression rate. KaplanMeier estimates were used to determine whether the Cyr61 expression is a potential prognostic factor for survival of these patients.

\section{Results}

Differences in Cyr61, Vim and E-cad expressions between primary tumors and tumor-adjacent normal tissues

The expressions of Cyr61, Vim and E-cad in LSCC are shown in Figure 1. Cyr61 positive staining was in tan or brown color and mainly localized in the cytoplasm. Vim positive staining was in brown color and localized in the intercellular substances. E-cad positive staining was brownish yellow and mainly localized in the cytoplasm.

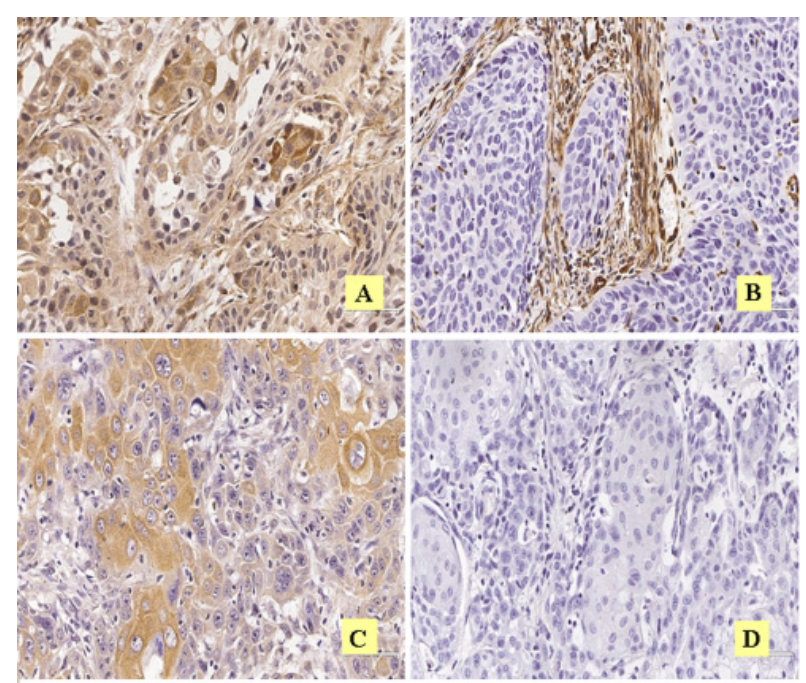

Figure 1. Expressions of Cyr61, Vim and E-cad in LSCC tissues $\times \mathbf{2 0 0}$. A) Cyr61 positive staining was in tan or brown color and mainly localized in the cytoplasm; B) Vim positive staining was brown and mainly localized in the intercellular substances; C) E-cad positive staining was brownish yellow and mainly localized in the cytoplasm. D: Negative control (PBS)

Table 1. Differences in Cyr61, Vim, and E-cad Expressions between the Primary Tumours and Tumour-adjacent Normal Tissues

\begin{tabular}{|c|c|c|c|c|c|c|c|}
\hline Indicator & Group & $\mathrm{N}$ & Positive(n) & Negative (n) & Positive rate $(\%)$ & $\chi^{2}$ & $\mathrm{P}$ \\
\hline \multirow[t]{2}{*}{ Cyr61 } & Primary tumour & 88 & 61 & 27 & 69.32 & 12.088 & 0 \\
\hline & Tumour-adjacent normal tissue & 30 & 10 & 20 & 33.33 & & \\
\hline \multirow[t]{2}{*}{ Vim } & Primary tumour & 88 & 68 & 20 & 77.27 & 9.885 & 0.002 \\
\hline & Tumour-adjacent normal tissue & 30 & 14 & 16 & 46.67 & & \\
\hline \multirow[t]{2}{*}{ E-cad } & Primary tumour & 88 & 29 & 59 & 32.95 & 8.556 & 0.003 \\
\hline & Tumour-adjacent normal tissue & 30 & 19 & 11 & 63.33 & & \\
\hline
\end{tabular}

Differences in the expression rates of Cyr61, Vim and E-cad between primary tumors and tumor-adjacent normal tissues are shown in Table 1. In primary tumors, the Cyr61 and Vim expression rates were higher whereas the E-cad expression rate was lower than those of the tumoradjacent normal tissues. The differences were statistically significant $(p<0.01)$.

Differences in Cyr61, Vim and E-cad expressions in primary tumors of different stages, primary sites, and degrees of differentiation

In primary tumors of different stages, primary sites and degrees of differentiation, differences in Cyr61, Vim and E-cad expressions are shown in Table 2-4. In Stage T3 and T4 LSCC, the Cyr61 and Vim expression rates were higher and the E-cad expression rate was lower than in stages T1 and T2 LSCC. The differences were statistically significant $(p<0.01)$. The Cyr61 and Vim expression rates were higher and the E-cad expression rate was lower in the group with lymph node metastasis $(\mathrm{N}+)$ than in the group without lymph node metastasis (N0). The differences were statistically significant $(p<0.05)$. The Vim expression rate was significantly higher in supraglottic carcinoma than in glottic carcinoma $(p<0.05)$. The E-cad expression rate was significantly higher in highly differentiated than in moderately and poorly differentiated primary tumors $(p<0.05)$. The Cyr61 expression rate did not show a significant correlation with the primary sites or degrees of differentiation of LSCC $(\mathrm{P}>0.05)$.

The correlation between Vim and E-cad expressions and Cyr61 expression

Based on the expression of Cyr61, primary tumors were divided into two groups. The correlation between the Cyr61 expression and Vim and E-cad expressions was investigated and the results are shown in Table 5. The Vim expression rate was higher whereas the E-cad expression rate was lower in the Cyr61 positive group than in the Cyr61 negative group. The differences were statistically significant $(p<0.05)$.

The correlation between Cyr61, Vim and E-cad expressions and LSCC prognosis

By using Kaplan-Meier method, survival analysis was performed on follow-up data of patients who were divided into different groups based on the expression levels of Cyr61, Vim and E-cad, and results are shown in Figure 2. The five-year survival rate of the Cyr61 positive group was significantly lower than that of the Cyr61 negative group $(p<0.001)$. The five-year survival rate of the Vim 
Table 2. Expression of Cyr61 in LSCC

\begin{tabular}{llccccc}
\hline Group & $\mathrm{N}$ & Positive $(\mathrm{n})$ & Negative $(\mathrm{n})$ & Positive rate $(\%)$ & $\chi^{2}$ & $P$ \\
\hline T1+T2 & 62 & 37 & 25 & 59.68 & 9.171 & 0.002 \\
T3+T4 & 26 & 24 & 2 & 92.31 & & \\
N0 & 59 & 36 & 23 & 61.02 & 5.801 & 0.016 \\
N+ & 29 & 25 & 4 & 86.21 & & \\
Glottic & 35 & 23 & 12 & 65.71 & 0.129 & 0.719 \\
Supraglottic & 53 & 38 & 15 & 71.7 & & \\
Highly differentiated & 60 & 41 & 19 & 68.33 & 0.086 & 0.769 \\
Moderately or poorly differentiated & 28 & 20 & 8 & 71.43 & & \\
\hline
\end{tabular}

Table 3. Expression of Vim in LSCC

\begin{tabular}{llccccc}
\hline Group & $\mathrm{N}$ & Positive $(\mathrm{n})$ & Negative $(\mathrm{n})$ & Positive rate $(\%)$ & $\chi^{2}$ & $P$ \\
\hline T1 + T2 & 62 & 43 & 19 & 69.35 & 7.491 & 0.006 \\
T3 + T4 & 26 & 25 & 1 & 96.15 & & \\
N0 & 59 & 39 & 20 & 66.1 & 12.722 & 0 \\
N+ & 29 & 29 & 0 & 100 & & \\
Glottic & 35 & 23 & 12 & 65.71 & 4.421 & 0.036 \\
Supraglottic & 53 & 45 & 8 & 84.91 & & \\
Highly differentiated & 60 & 48 & 12 & 80 & 0.799 & 0.372 \\
Moderately or poorly differentiated & 28 & 20 & 8 & 71.43 & & \\
\hline
\end{tabular}

\section{Table 4. Expression of E-cad in LSCC}

\begin{tabular}{lcccccc}
\hline Group & $\mathrm{N}$ & Positive $(\mathrm{n})$ & Negative $(\mathrm{n})$ & Positive rate (\%) & $\chi^{2}$ & $P$ \\
\hline T1 + T2 & 62 & 28 & 34 & 45.16 & 14.152 & 0 \\
T3 + T4 & 26 & 1 & 25 & 3.85 & & \\
N0 & 59 & 26 & 33 & 44.07 & 10.008 & 0.002 \\
N+ & 29 & 3 & 26 & 10.34 & & \\
Glottic & 35 & 13 & 22 & 37.14 & 0.461 & 0.497 \\
Supraglottic & 53 & 16 & 37 & 30.19 & & \\
Highly differentiated & 60 & 25 & 35 & 41.67 & 6.478 & 0.011 \\
Moderately or poorly differentiated & 28 & 4 & 24 & 14.29 & & \\
\hline
\end{tabular}

Table 5. Correlations between the Expression of Vim, E-cad, and Cyr61

\begin{tabular}{|c|c|c|c|c|c|c|c|}
\hline Indicator & Group & $\mathrm{N}$ & Positive (n) & Negative(n) & Positive rate $(\%)$ & $\chi^{2}$ & $\mathrm{P}$ \\
\hline \multirow[t]{2}{*}{ Vimentin } & Cyr61(+) & 61 & 51 & 10 & 83.61 & 4.542 & 0.033 \\
\hline & Cyr61(-) & 27 & 17 & 10 & 62.96 & & \\
\hline \multirow[t]{2}{*}{ E-cadherin } & Cyr61(+) & 61 & 15 & 46 & 24.59 & 6.296 & 0.012 \\
\hline & Cyr61(-) & 27 & 14 & 13 & 51.85 & & \\
\hline
\end{tabular}
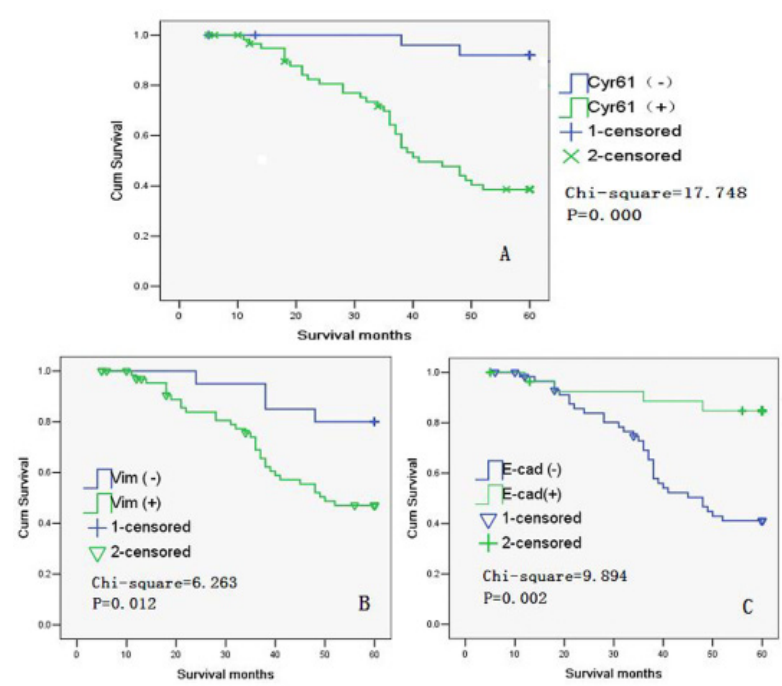

Figure 3. Correlation between Cyr61, Vim and E-cad Expressions and Survival Rates (A: Cyr61, B: Vim, C: E-cad) positive group was significantly lower than that of the Vim negative group $(p<0.05)$. However, the five-year survival rate was significantly higher in the E-cad positive group than in the E-cad negative group $(p<0.01)$.

\section{Discussion}

Cyr61/CCN1 is a cysteine-rich secretory protein and was first identified by Lau et al. in 1985. It was the first cloned gene in the CCN family (CCN1-CCN6) ( Lau et al., 1985). Cyr61 is an important regulatory factor of ECM located in downstream of many intracellular and extracellular signal transduction pathways, regulating the adhesion, migration and proliferation of endothelial cells and fibroblasts, inducing angiogenesis, and involved in processes such as inflammatory responses, wound healing and tissue remodeling (Dhar et al., 2010; Zuo et al., 2010). It also plays different roles in tumor initiation and progression (Bleau et al., 2005). Cyr61 expression is increased in rhabdomyosarcoma, malignant melanoma, 
colon cancer, bladder cancer, and pancreatic cancer (Genini et al., 1996; Schmitz et al., 2013; Maity et al., 2014). In giiomas, Cyr61 is highly expressed through the integrin-linked kinase signaling pathway (Sin et al., 2008). Cyr61 is overexpressed in breast cancer (Xie et al., 2001), inducing tumor cell proliferation and migration, hence playing an oncogenic role. The expression of Cyr61 is upregulated in prostate cancer and esophageal squamous cell carcinoma (Xie et al., 2011; Lin et al., 2014), but downregulated in lung cancer. Cyr61 inhibits lung cancer, endometrial cancer and leiomyoma by mediating tumor cell apoptosis and inhibiting tumor cell growth, hence exerting an antitumor effect (Sampath et al., 2001; Chien et al., 2004; Mori et al., 2007). The inconsistent expression of Cyr61 in different tumor tissues suggests that Cyr61 plays multiple roles, and has strong functions of regulating and being regulated by other genes.

Cyr61 is a ligand for several integrins including $\alpha v \beta 3$, $\alpha v \beta 5, \alpha \operatorname{IIb} \beta 3, \alpha \mathrm{M} \beta 2$ and $\alpha \mathrm{D} \beta 2$, and its interaction with integrins regulates different cell functions through a variety of cell signaling pathways (Chen et al., 2009). Cyr61 binding to $\alpha v \beta 3$ and $\alpha v \beta 5$ mediates the adhesion and migration of endothelial cells, and plays a key role in angiogenesis (Jandova et al., 2012). Cyr61 binding to $\alpha v \beta 3$ and/or $\alpha v \beta 5$ not only results in its biological effectiveness, but also further stimulates the secretion of $\alpha v \beta 3$ and $\alpha v \beta 5$, forming a Cyr61- $\alpha v \beta 3 / \alpha v \beta 5$ autocrine regulatory loop and producing cell growth and antiapoptotic signals (Menendez et al., 2005). The $\alpha \mathrm{v}$ integrin family is a type of RGD receptor and a natural activator of the inactive TGF- $\beta$ precursor. Overexpression of $\alpha \mathrm{v}$ integrins activates the TGF- $\beta$ signal pathway triggering a series of subsequent biological effects (Roth et al., 2013). TGF- $\beta$ is an essential inducing factor for virtually all EMTs occurring in the epithelial tissues. TGF- $\beta$ activation and overexpression are able to stimulate Cyr61 secretion in tumor cells (Bartholin et al., 2007). Therefore we speculate that there might be a complete autocrine regulatory loop between Cyr61, $\alpha$ v integrins, and TGF- $\beta$, and tumor cells are likely self-regulating EMT initiation through this loop.

LSCC is a common clinical malignant tumor and poses a serious threat to human life and health. To our knowledge, studies on the expression and biological function of Cyr61/CCN1 in LSCC have not been reported. In this study, we used immunohistochemistry technique to detect Cyr61/CCN1 expression in 88 cases of LSCC and 30 cases of tumor-adjacent tissues, as well as the expressions of the epithelial marker E-cad and mesenchymal marker Vim. The results indicated that in LSCC, the Vim expression rate was significantly higher and the E-cad expression rate was significantly lower compared to the tumor-adjacent tissues. The expression rate of Vim was significantly higher in stages T3 and T4 than in stage T1 and T2 LSCC, whereas the E-cad expression rate was significantly lower in stage $\mathrm{T} 3$ and $\mathrm{T} 4$ than in stages T1 and T2 LSCC. The Vim expression rate was higher whereas the E-cad expression rate was lower in the group with lymph node metastasis than in the group without lymph node metastasis. The differences were statistically significant $(p<0.01)$. The results suggested that the EMT clearly occurred in the process of LSCC cell invasion and migration. As an important regulatory factor, Cyr61/CCN1 might play an important role in this process. Our results indicated that the Cyr61/CCN1 expression rate was significantly higher in LSCC than in tumor-adjacent tissues, and was closely associated with LSCC clinical stages, lymph node metastasis and prognosis. More importantly, in LSCC with high expression of Cyr61, the expression rate of mesenchymal marker Vim was higher and expression rate of the epithelial marker E-Cad was lower compared to the group with low expression of Cyr61, and the differences were statistically significant $(p<0.01)$, indicating that high expression of Cyr6 likely induced the EMT of LSCC cells.

In this study, we showed on protein expression level that overexpression of Cyr61 is closely associated with LSCC invasion and metastasis and prognosis. In addition, based on differences in expression levels of the epithelial marker E-cad and the mesenchymal maker Vim, we speculated that Cyr61 overexpression was associated with the EMT of the LSCC. In order to further confirm the function of Cyr61 expression in regulating LSCC biological behaviors, and whether the Cyr61/ $\alpha v \beta 5$ autocrine regulatory loop does exist in LSCC cells and plays an important regulatory role in growth and proliferation, additional studies using molecular biology and cell biology methods are required. Cyr61 is a multifunctional regulatory factor and plays an important role in LSCC invasion and metastasis, and therefore it may be a new molecular maker and important treatment target of LSCC, which is worth further studies.

\section{References}

Bartholin L, Wessner LL, Chirgwin JM, Guise TA (2007). The human Cyr61 gene is a transcriptional target of transforming growth factor beta in cancer cells. Cancer Lett, 246, 230-6.

Baum B, Settleman J, Quinlan MP (2008). Transitions between epithelial and mesenchymal states in development and disease. Semin Cell Dev Biol, 19, 294-308.

Bleau AM, Planque N, Perbal B (2005). CCN proteins and cancer: two to tango. Front Biosci, 10, 998-1009.

Chen C, Zimmermann M, Tinhofer I, Kaufmann AM, Albers $\mathrm{AE}$ (2013). Epithelial-to-mesenchymal transition and cancer stem (-like) cells in head and neck squamous cell carcinoma. Cancer Lett, 338, 47-56.

Chen CC, Lau LF (2009). Functions and mechanisms of action of CCN matricellular proteins. Int J Biochem Cell Biol, 41, 771-83.

Chien W, Kumagai T, Miller CW, et al (2004). Cyr61 suppresses growth of human endometrial cancer cells. J Biol Chem, 279, 53087-96.

Cichon MA, Radisky DC (2014). Extracellular matrix as a contextual determinant of transforming growth factor-b signaling in epithelial-mesenchymal transition and in cancer. Cell Adh Migr, 8, 588-94.

Crockett JC, Schütze N, Tosh D, et al (2007). The matricellular protein CYR61 inhibits osteoclastogenesis by a mechanism independent of alphavbeta3 and alphavbeta5. Endocrinology, 148, 5761-8.

Dhar A, Ray A(2010). The CCN family proteins in carcinogenesis. Exp Oncol, 32, 2-9.

Genini M, Schwalbe P, Scholl FA, Schafer BW (1996). Isolation of genes differentially expressed in human primary myoblasts and embryonal rhabdomyosarcoma. Int J Cancer, 
66, 571-7.

Grant CM, Kyprianou N (2013). Epithelial mesenchymal transition (EMT) in prostate growth and tumor progression. Transl Androl Urol, 2, 202-11.

Hou CH, Lin FL, Hou SM, Liu JF (2014). Cyr61 promotes epithelial-mesenchymal transition and tumor metastasis of osteosarcoma by Raf-1/MEK/ERK/Elk-1/TWIST-1 signaling pathway. Mol Cancer, 13, 236.

Jandova J, Beyer TE, Meuillet EJ, Watts GS (2012). The matrix protein $\mathrm{CCN} 1 / \mathrm{CYR} 61$ is required for $\alpha(\mathrm{V}) \beta 5$-mediated cancer cell migration. Cell Biochem Funct, 30, 687-95.

Juric V, Chen CC, Lau LF (2012). TNF $\alpha$-induced apoptosis enabled by CCN1/CYR61: pathways of reactive oxygen species generation and cytochrome c release. PLoS One, 7, 31303

Lau LF, Nathans D (1985). Identification of a set of genes expressed during the G0/G1 transition of cultured mouse cells. EMBO J, 4, 3145-51.

Lau LF (2011). CCN1/CYR61: the very model of a modern matricellular protein. Cell Mol Life Sci, 68, 3149-63.

Leu SJ, Chen N, Chen CC, et al (2004). Targeted mutagenesis of the angiogenic protein CCN1 (CYR61). Selective inactivation of integrin alpha6beta1-heparan sulfate proteoglycan coreceptor-mediated cellular functions. J Biol Chem, 279, 44177-87.

Lin CM, Liang CZ (2014). Cyr61: a potential therapeutic target for prostate cancer. Asian J Androl, 16, 788-9.

Lu JG, Li Y, Li L, Kan X (2011). Overexpression of osteopontin and integrin $\alpha \mathrm{v}$ in laryngeal and hypopharyngeal carcinomas associated with differentiation and metastasis. J Cancer Res Clin Oncol, 137, 1613-8.

Maity G, Mehta S, Haque I, et al (2014). Pancreatic tumor cell secreted CCN1/Cyr61 promotes endothelial cell migration and aberrant neovascularization. Sci Rep, 4, 4995.

Menendez JA, Vellon L, Mehmi I, et al (2005). A novel CYR61triggered 'CYR61-alphavbeta3 integrin loop' regulates breast cancer cell survival and chemosensitivity through activation of ERK1/ERK2 MAPK signaling pathway. Oncogene, 24, 761-79.

Monnier Y, Farmer P, Bieler G, et al (2008). CYR61 and alphaVbeta5 integrin cooperate to promote invasion and metastasis of tumors growing in preirradiated stroma. Cancer Res, 68, 7323-31.

Mori A, Desmond JC, Komatsu N, et al (2007). CYR61: a new measure of lung cancer outcome. Cancer Invest , 25, 738-41.

Roth P, Silginer M, Goodman SL, et al (2013). Integrin control of the transforming growth factor- $\beta$ pathway in glioblastoma. Brain, 136, 564-76.

Sampath D, Zhu Y, Winneker RC, Zhang Z (2001). Aberrant expression of Cyr61, a member of the CCN (CTGF/Cyr61/ Cef10/NOVH) family, and dysregulation by 17 betaestradiol and basic fibroblast growth factor in human uterine leiomyomas. J Clin Endocrinol Metab, 86, 1707-15.

Schmitz P, Gerber U, Schütze N, et al (2013). Cyr61 is a target for heparin in reducing MV3 melanoma cell adhesion and migration via the integrin VLA-4. Thromb Haemost, 110, 1046-54.

Sin WC, Bechberger JF, Rushlow WJ, Naus CC (2008). Dosedependent differential upregulation of CCN1/Cyr61 and $\mathrm{CCN} 3 / \mathrm{NOV}$ by the gap junction protein Connexin43 in glioma cells. J Cell Biochem, 103, 1772-82.

Su JL, Chiou J, Tang CH, et al (2010). CYR61 regulates BMP2-dependent osteoblast differentiation through the \{alpha\} $\mathrm{v}$ \{beta\}3 integrin/integrin-linked kinase/ERK pathway. $J$ Biol Chem, 285, 31325-36.

Sung YK, Kwack MH, Kim SR, Kim MK, Kim JC (2008). Transcriptional activation of $\mathrm{CCN} 1$ and CCN2, targets of canonical Wnt signal, by ascorbic acid 2-phosphate in human dermal papilla cells. J Dermatol Sci, 49, 256-9.

Tabatabai G, Moch H, Tritschler I, et al (2013). Integrin control of the transforming growth factor- $\beta$ pathway in glioblastoma. Brain, 136, 564-76.

Todorovic V, Chen CC, Hay N, Lau LF (2005). The matrix protein CCN1 (CYR61) induces apoptosis in fibroblasts. $J$ Cell Biol, 171, 559-68.

Tsai MS, Bogart DF, Castañeda JM, Li P, Lupu R (2002). Cyr61 promotes breast tumorigenesis and cancer progression. Oncogene, 21, 178-85.

Wang Y, Zhou BP (2013). Epithelial-mesenchymal Transition-a hallmark of breast cancer metastasis. Cancer Hallm, 1, 38-49.

Xie D, Miller CW, O'Kelly J, et al (2001). Breast cancer. Cyr61 is overexpressed, estrogen-inducible, and associated with more advanced disease. J Biol Chem, 276, 14187-194.

Xie JJ, Xu LY, Xie YM, et al (2011). Involvement of Cyr61 in the growth, invasiveness and adhesion of esophageal squamous cell carcinoma cells. Int J Mol Med, 27, 429-34.

Yang J, Weinberg RA (2008). Epithelial-mesench;ymal transition: at the crossroads of development and tumor metastasis. Dev Cell, 14, 818-29.

Zuo GW, Kohls CD, He BC, et al (2010). The CCN proteins: important signaling mediators in stem cell differentiation and tumorigenesis. Histol Histopathol, 25, 795-806. 